\title{
Perspectivas Ergológicas para o Uso de Simuladores de Direção
}

\author{
Thiago Drumond Moraes ${ }^{1}$ \\ Departamento de Psicologia Social e do Desenvolvimento da Universidade Federal \\ do Espirito Santo, Vitória, ES, Brasil \\ Yves Schwartz \\ Institute d'Ergologie, Université d'Aix-Marseille, Aix-en-Provence, França \\ Centre d'EPistémologie et d'ERgologie Comparatives - Centre National \\ de la Recherche Scientifique, Aix-en-Provence, França
}

\begin{abstract}
Resumo
Apesar da controversa legislação brasileira sobre formação por simuladores de direção, seu uso ainda é escasso. Além disso, não há obrigatoriedade de seu emprego em formação de condutores rodoviários profissionais. Segundo a literatura internacional, onde o assunto é estudado há mais tempo, ainda não há evidência suficiente que justifique o amplo uso de simuladores em formações de condutores profissionais. Por outro lado, não se observa nessa literatura uma análise crítica dos efeitos dos fundamentos teórico-metodológico utilizadas nessas formações. Visando contribuir para esse debate, revisamos a literatura sobre uso de simuladores em formações de condutores rodoviários profissionais, analisando-a criticamente a partir das contribuições da Ergologia e da Didática Profissional francesa. Observamos problemas nas formações experimentadas: (a) não reconhecimento da atividade real a simular e dos saberes socialmente construídos necessários para executá-la; (b) concepção insuficiente sobre competências e saberes mobilizados em situação de trabalho; (c) incapacidade de apreensão de uma atividade profissional. Apresentamos alternativas para uso de simuladores nas formações de condutores profissionais: formações coletivas mediadas pela simulação e em torno de saberes, normas e valores partilhados pelos condutores.
\end{abstract}

Palavras-chave: Simuladores de direção, transporte, formação profissional, Ergologia.

\section{Ergological Perspectives for the Use of Driving Simulators}

\begin{abstract}
Despite controversial Brazilian legislation on simulation-based driver training, it is used only rarely. Furthermore, there is no requirement to use it in professional road driver training. According to the international literature, in which the subject has been studied for a much longer period, there is still insufficient evidence to justify the widespread use of simulators to train professional drivers. However, this literature contains no critical analysis of the effects of the theoretical and methodological foundations utilised in this training. To contribute to this debate, we review the literature on simulation-based

Endereço para correspondência: Universidade Federal do Espírito Santo, Departamento de Psicologia Social e do Desenvolvimento, Cemuni VI, campus Goiabeiras, Av. Fernando Ferrari, 514, Goiabeiras, Vitória, ES, Brasil 29075-910. Fone: (27) 4009-2505. E-mail: thiago.moraes@ufes.br e yves.schwartz@univ-amu.fr Financiador Coordenação de Aperfeiçoamento de Pessoal de Nível Superior - CAPES (Thiago Drumond Moraes Bolsista, processo n. BEX 2103/14-1) e Fundação de Amparo à Pesquisa do Espírito Santo (FAPES).
\end{abstract}


training for professional drivers, critically analysing it through the contributions of Ergology and French Professional Didactics. We note some problems with the different training programmes examined: (a) no recognition of the actual activity being simulated or the socially constructed knowledge that is necessary to perform it; (b) an insufficient conception of the skills and knowledge mobilised in work situations; and (c) a failure to understand a professional activity. We present alternative methods of using simulation-based training for professional drivers: collective training mediated by simulation and based on the knowledge, norms, and values shared by drivers.

Keywords: Driving simulators, transport, professional training, Ergology.

\section{Perspectivas Ergológicas para el Uso de Simuladores de Conducción}

\section{Resumen}

A pesar de polémica legislación brasileña sobre formación de simuladores de conducción, su uso es escaso. Además, no se obliga su uso en formación de conductores profesionales. En la literatura internacional, donde el tema es pesquisado hace mucho tiempo, no existe evidencia suficiente para justificar el uso generalizado de simuladores en formaciones de conductores profesionales. Sin embargo, no se observa en esta literatura un análisis crítico de los efectos de los fundamentos teóricos y metodológicos utilizadas en estas formaciones. Contribuyendo a este debate, se revisa la literatura sobre uso de simuladores en formación de conductores profesionales, analizando-as críticamente a partir de contribuciones de la Ergología y de la Didáctica Profesional francesa. Observamos problemas en la formación pesquisadas: (a) no reconocimiento de la actividad para simular y de los saberes socialmente construidos necesario para la realizar la actividad; (b) concepción insuficiente sobre saberes y competencias movilizados en situaciones de trabajo; (c) incapacidad de aprehender una actividad professional. Presentamos alternativas para uso de simuladores en formación de conductores profesionales: formaciones colectivas mediadas por simulación y alrededor de saberes, normas y valores compartidos por los conductores.

Palabras clave: Simuladores de conducción, transporte, formación profesional, Ergologia.

Formações profissionais são realizadas por meio de diversas estratégias metodológicas, entre as quais a simulação (Abbad, Zerbini, Carvalho, \& Meneses, 2006). Embora não seja recente, o uso dessa estratégia nos mais variados campos da formação profissional, entre as quais os trabalhadores dos transportes terrestres, foi impulsionado pelas novas tecnologias de informação (Blanco, Hickman, Hanowski, \& Morgan, 2011).

São variadas as maneiras de se conceber e utilizar as simulações, refletindo os pressupostos teóricos embasando tais usos (Pottier, 2013). As simulações são frequentemente acompanhadas de suportes e instrumentos tecnológicos, usualmente concebidos como simuladores. Apesar de se valorizar a versatilidade desse instrumento (Blanco et al., 2011), frequentemente se reduz os usos dos simuladores e da simulação para treinamentos com estilo de aprendizagem corporal-cinestésica (Castro \& Ferreira, 2006).

$\mathrm{O}$ uso de simulação e simuladores vem ganhando espaço privilegiado em várias áreas da formação profissional, porém talvez seja no campo da saúde e na formação de pilotos de avião que se encontrem os terrenos mais bem consolidados de aplicação de simuladores (Goode, Salmon, \& Lenné, 2013). Em contrapartida, no campo dos transportes rodoviários, setor responsável pela maior quantidade de mortes em acidentes de trabalho em muitos países desenvolvidos e em desenvolvimento (Mooren, Grzebieta, Williamson, Olivier, \& Friswell, 2014; Santana, Nobre, \& Waldvogel, 2005), o uso de simuladores profissionais ainda não se encontra generalizado, a despeito do seu 
crescimento recente (Blanco et al., 2011; Moraes, 2016).

Entre as razões para que não se utilizar simuladores em formações de trabalhadores dos transportes rodoviários de maneira generalizada estão a falta de evidências científicas sobre seus benefícios, o alto custo da aquisição desses instrumentos sem retorno financeiro comprovado e escassez de instrutores qualificados para usar, de modo efetivo, as técnicas de simulação e operar adequadamente os simuladores (Grüneberg \& Schröder, 2012). Essas reflexões são frutos de pesquisas sobre usos e apropriações legislativas desses instrumentos na_formação profissional há quase 15 anos em países desenvolvidos (Directive 2003/59/EC, 2003). No Brasil, só recentemente assiste-se a um debate político sobre a introdução sistemática desse tipo de tecnologia na formação para condutores de transporte rodoviário (Resolução Conselho Nacional de Trânsito $n^{0}$ 493, 2014; Resolução Conselho Nacional de Trânsito $\left.n^{0} 543,2015\right)$. Além de indicar o uso opcional de simuladores na formação de condutores em geral, a resolução nacional nada diz sobre a formação de condutores profissionais. Dever-se-ia elaborar resoluções semelhantes para estes últimos, tal qual a resolução europeia mencionada?

De fato, não se observa no campo da formação de trabalhadores para os transportes rodoviários o mesmo sucesso no uso de simuladores que se verifica na formação de trabalhadores da aviação e da saúde. Daí se questionar as razões de tal relativo fracasso, ressaltando-se, inclusive, aspectos econômicos (Grüneberg \& Schröder, 2012; Moraes, 2016). Entretanto, além dos fatores citados por esses autores, demonstraremos outro que limita os possíveis benefícios do uso de simuladores para a formação de condutores profissionais: os usos correntes desse instrumento são limitados pelas concepções teóricas que os embasam, o que contribui para reduzir seus efeitos. Para auxiliar na avaliação sobre a (in) adequação do uso dos simuladores nas formações profissionais, realizamos a análise da literatura internacional sobre a formação profissional por meio desses instrumentos, investigando as concepções teóricas utilizadas nessas formações e em que medida elas ampliam ou limitam seus impactos. Para tanto, analisamos criticamente os modos como eles vêm sendo empregados nas formações em países onde tais práticas são mais antigas e/ou comuns.

Realizamos a análise da literatura embasados nas perspectivas da Ergologia (Schwartz, 2012; Schwartz \& Durrive, 2010) e da Didática Profissional (Pastré, 2011). Ambas partilham algumas concepções que nos são cruciais para se compreender as potencialidades do uso de simuladores: (a) que em toda atividade profissional se produzem saberes socialmente partilhados, mais ou menos relacionados aos saberes socialmente instituídos e, (b) que estes saberes se constituem pelo engajamento ativo dos trabalhadores, com sua história, seus debates de normas e seus valores, na prática profissional em situações concretas de trabalho. É, portanto, na atividade, e senão por meio dela, que se aprende. Sem levar isso em conta, a eficácia do uso de simuladores estará limitada apenas a situações muito específicas, tais como às situações de laboratório.

Para realizar a análise da literatura, lançamos mão de textos publicados em revistas científicas, em anais de congressos temáticos, em livros e em relatórios de pesquisas financiadas por órgãos governamentais e não governamentais. Apresentaremos a seguir um consolidado dos principais resultados encontrados e, posteriormente, algumas análises críticas mobilizadas pelas referências teóricas citadas. Ressaltamos que doravante, o uso do termo simuladores referir-se-á a simuladores de direção elaborados para condutores profissionais.

\section{Principais Resultados Encontrados em Publicações sobre Formação Profissional com Usos de Simuladores de Direção}

A maioria dos estudos foram realizados com simuladores de base fixa, visão de $180^{\circ}$ a $270^{\circ}$, retrovisores virtuais e comandos realísticos (volante, marchas, pedais, etc.). Entretanto, os resultados obtidos nas pesquisas foram menos relacionados ao tipo de simulador utilizado e mais à relação entre as características que eles 
permitem treinar e os desenhos das formações (Brock, Jacobs, Van Cott, McCauley, \& Norstrom, 2001; Moraes, 2016).

Os estudos laboratoriais de desenvolvimento e teste de simuladores atestam capacidade deles propiciarem aprendizagens de algumas das competências, como controle básico do veículo, manobras de estacionamento para descarga e economia de combustível (Dorn \& Stannard, 2006; Uhr, Felix, Williams, \& Krueger, 2003). Em estudos de qualidade variável, verificam-se indícios de que a interação homem-máquina propicia aprendizagens, mas que estas melhoram se a formação for mais customizada (Tarr, 2005) e se os instrumentos forem mais adequados a seus objetivos (Tarr \& Whitmire, 2008). Uma vez que tais estudos laboratoriais não foram replicados em situação real, poderíamos questionar sua validade para orientar o uso de simuladores em treinamentos reais. Entretanto, há estudos que avaliaram a eficácia de treinamento com simuladores em organizações reais, confirmando parcialmente a potencialidade dessas formações substituírem e/ou complementarem as tradicionais formações on-the-road (em situações reais) e behind-the-wheel (em veículos reais), seja por estes apresentarem desempenho similar na transferência de aprendizagem (Morgan et al., 2011), pela avaliação positiva dos usuários (Neukum, Lang, \& Krueger, 2003; Reed, Parkes, Peacock, Lang, \& Rehm, 2007), por propiciarem uma aprendizagem mais veloz (Lindsey \& Barron, 2008) ou por demonstrarem efetividade na transferência de competências, sobretudo no que se refere à aprendizagem de economia de combustível (Reed et al., 2007; Strayer \& Drews, 2003). Por outro lado, por não serem estudos longitudinais, os efeitos do uso de simuladores em tais treinamentos, ao longo do tempo, ainda está por ser investigado.

No que tange às avaliações de programas de formação em uso regular pelas empresas transportadoras de produtos e/ou pessoas, demonstrou-se que tais treinamentos são avaliados positivamente pelos usuários, sejam eles aprendizes (Aginaga-Garcia, Iriarte-Goni, Pintor-Borobia, Ros-Ganuza, \& San Miguel-Indurain, 2013), instrutores ou diretores das indústrias de trans- portes rodoviários (Brock et al., 2001; Reep, Staes, \& Perk, 2013). Observou-se também que em casos de treinamento para economia de combustível, houve ganho de desempenho econômico da condução, com melhora de 4,5\% a 6,1\% (Kihl, Herring, Wolf, Finn, \& Yang, 2007), que para os aprendizes a resposta simulada dos comandos é mais importante que o quanto tais comandos se assemelham fisicamente aos comandos reais (Brook-Carter, Luke, \& Parkes, 2004) e que o tipo de simulador pode influenciar no desempenho de aprendizagem, exigindo que as empresas estejam atentas a que competências treinar, antes de adquirir simuladores para a formação (Brock et al., 2001). Não há evidências que o treinamento com esses instrumentos contribua para redução de acidentes (Goode et al., 2013; Grüneberg \& Schröder, 2012), embora uma publicação, sem verificação científica segura, sugira que esse tipo de formação reduziu acidentes em 5,9\% (Edson, 2003). Destacam-se dessas publicações indícios de transferências de aprendizagens para situações reais, mas tais indícios não encontram sustentação sólida em dados objetivos ou inquestionáveis, dadas as dificuldades de avaliação impostas pelos registros deficitários das empresas, não habituadas a registros cuidadosos dessas informações (Kihl, Herring, Wolf, McVey, \& Kovuru, 2006).

Em suma: os simuladores são instrumentos potencialmente interessantes, mas cujo conjunto de textos investigados não demonstrou, com evidência suficiente, que é um instrumento melhor, mais adequado e economicamente viável que as formações tradicionais de condutores profissionais (Moraes, 2016). Grüneberg e Schröder (2012) ressaltam que apesar de interessante, ainda não há dados que comprovam compensação pelo pesado investimento de aquisição, preparação e manutenção de simuladores para treinamentos, o que lança sombras sobre seu futuro. Por outro lado, estudos nos EUA demonstraram grandes companhias de transporte de cargas fazendo uso sistemático desses instrumentos (Morgan et al., 2011), apesar de que tal pesquisa refere-se a período anterior à crise imobiliária de 2008; crise essa que pode ter alterado este quadro. 


\section{Usos dos Simuladores nas Formações Profissionais}

Na medida em que uma prática de formação deve estar ancorada em teorias sobre aprendizagem, saberes e atividade e que tais teorias influem nos diferentes usos que se faz de simuladores (Pottier, 2013), torna-se relevante averiguar quais teorias foram referenciadas na literatura. Mesmo que poucos textos ressaltem os embasamentos teóricos que orientam suas práticas, uma análise cuidadosa nos permitiu deduzir alguns de seus fundamentos.

\section{Competências Avaliadas}

São diversas as competências treinadas e/ou averiguadas pelos simuladores. As mais frequentemente investigadas referem-se ao manejo do veículo. Tratam-se de treinamentos para novatos aprenderem o controle básico (manter o veículo na pista, negociar curvas, estacionar em ré, em situações difíceis ou com cargas especiais) ou para profissionais novatos e experientes desenvolverem controle avançado do veículo, tais como reação adequadas em situações raramente vivenciadas (p. ex. estouro de pneus; Morgan et al., 2011) ou atualizações para ação em climas específicos e sazonais (reinício da temporada de inverno para condutores de removedores de neve; Kihl et al., 2007; Strayer \& Drews, 2003; Tarr, 2005). Soma-se a esse controle a necessidade de conduzir de maneira segura (direção segura ativa e passiva) e econômica (direção ecológica e economicamente eficiente). As resoluções brasileiras sobre simuladores de direção também se referem a essas mesmas competências.

De modo geral, os simuladores tendem a ser concebidos como substitutos de um veículo real para treinamento cinestésico-corporal do condutor (Moraes, 2016; Morgan et al., 2011). São pouco investigadas competências menos relacionadas ao manejo do veículo, mas que fazem parte das tarefas de condução profissional, tais como 'tarefas de comunicação', 'competências coletivas não técnicas', 'tarefas extra-volante' e 'planejamento geral da condução e das tarefas a serem realizadas'. Tais tarefas, menos necessá- ria para a aprendizagem de condutores regulares da categoria B, objeto das resoluções brasileiras, assumem um papel muito importante no caso de condutores profissionais (Valot, 2007).

Apenas dois estudos sugerem, sem demonstração empírica, que simuladores poderiam treinar aspectos psicomotores e afetivos envolvidos na condução (Bonch-Bruevich, Kremez, \& Chirkov, 2010; Navarrete, Urquiza, Hernandez, $\&$ Madrid, 2004). Por outro lado, estudos apresentam treinamentos que abordam atitudes dos condutores em relação à condução segura e/ou econômica, (Reed et al., 2007; Tarr \& Whitmire, 2008), o que sinaliza inclusão incipiente das reações afetivas nos programas de treinamento com simuladores. Há textos teóricos que indicam maior diversidade de competências que poderiam ser formadas pelos simuladores, porém sem apresentar estudos empíricos correlatos, o que é um indício da defasagem entre possibilidades do instrumento e experiências empíricas realizadas.

\section{Atitudes dos Usuários}

Aprendizes avaliam de modo distinto os simuladores em função de suas expertises. A importância e a satisfação em relação ao uso do equipamento em treinamentos foram maiores entre condutores menos experientes (Kihl et al., 2006; Morgan et al., 2011). Trabalhadores mais experientes avaliam como interessante as formações com simuladores, mas não as julgam compatível à realidade, seja pela falta de controle operacional dos simuladores, seja porque alguns controles operacionais não emitem pistas sensoriais necessárias para a gestão adequada do veículo (Kihl et al., 2006), ou porque os treinamentos superdimensionam a frequência de incidentes que, em situações reais, são relativamente raras, o que, segundo os trabalhadores,_torna a experiência de simulação razoavelmente irreal (Morgan et al., 2011). Ainda que as resoluções brasileiras supracitadas refiram-se à formação de condutores novatos, o planejamento de formações continuadas com condutores profissionais deve considerar tais avaliações de profissionais mais experientes, para tornar o uso de simuladores mais efetivo. 


\section{Pressupostos Teórico-Epistêmico- Metodológicos}

Poucos estudos mencionaram os pressupostos teórico-epistemológicos que embasam os usos, análises e desenvolvimento dos treinamentos com simuladores. Quando existente, tratam-se de referências breves a modelos que se referem mais a níveis comportamentais e cognitivos das competências, que às dimensões psicoafetivas e atitudinais da condução, tais quais os modelos de Rasmussen (1983; Blanco et al., 2011; Brock et al., 2001; Morgan et al., 2011; Victor et al., 2011), Michon (1985; Kihl et al., 2006; Neukum et al., 2003), e Bloom (Navarrete et al., 2004). Os modelos cognitivos de Michon e Rasmussem apresentam muitas características comuns, embora o primeiro tenha sido proposto para explicar o comportamento do condutor e o segundo para explicar o comportamento de operadores diante incidentes no trabalho. Ambos estabelecem 3 níveis de habilidades e controle dos veículos/máquinas: o nível de controle do veículo (Michon), ou das habilidades (Rasmussen), refere-se a padrões comportamentais automatizados, de resposta temporal na ordem de milissegundos, que visam garantir o controle operacional do veículo/máquina operando em nível pré-consciente e demandando poucos recursos cognitivos para garantir a solução dos incidentes; o nível de manobras (Michon) ou de regras (Rasmussen), refere-se a ações mais ou menos sequenciais e esquematizadas, disparadas quando há indícios de que os esquemas automáticos são parcialmente insuficientes para gerir as situações encontradas, ou na negociação do veículo com outros veículos nas vias, por meio de soluções mais ou menos padronizadas, requerendo mais recursos cognitivos e mais tempo que o nível anterior; e o nível estratégico (Michon) e de conhecimento (Rasmussen), que se referem ao planejamento das rotas e à busca de soluções ainda não necessariamente conhecidas ou automatizadas para problemas inesperados, e operam na ordem de um tempo longo e constante, demandando muitos recursos cognitivos. Observa-se que em ambos os modelos não se aborda a dimensão afetiva do condutor. Apenas no texto dos autores que se referiram à taxonomia de
Bloom, modelo mais geral sobre competências e aprendizagem humana, é que a dimensão afetiva é brevemente abordada. Chamou-nos também a atenção que nenhum dos textos discutiu aspectos sociais, culturais e os valores subjacentes aos comportamentos de condução, aspectos também não contemplados pelos principais modelos utilizados pelos estudos supracitados.

Igualmente, pouco se descreveu sobre os métodos utilizados. Apresenta-se, por vezes, o desenho geral do curso, geralmente sendo utilizado o desenho teórico-prático (salas de aula + simuladores), na seguinte sequência: sala de aula para introdução do assunto, exercício prático no simulador, seguido de avaliação do desempenho mediado pelo instrutor (Reed et al., 2007; Strayer \& Drews, 2003). Este sistema é mais comumente encontrado em treinamentos mais curtos e específicos para determinada competência. Em outros casos, alia-se ao treinamento com simuladores práticas em veículos reais (salas de aula + simuladores + veículos reais) (Aginaga-Garcia et al., 2013; Lindsey \& Barron, 2008; Morgan et al., 2011). Tal procedimento, preconizado também pelas resoluções brasileiras, é mais comum em formação de profissionais para o desempenho inicial da profissão. As diferenças de desenho de curso variam muito em função dos cenários a se simular. Em caso de cenários complexos, que mobilizam competências muito específicas e focadas para condutores experientes, e que envolvem situações de risco, por razões de segurança e viabilidade, tende-se a não se replicar em situações reais os procedimentos adotados em simuladores. Já no caso de cursos para iniciantes, dados indicam que o uso de veículos reais nas formações não é dispensável (Morgan et al., 2011). A possibilidade dos simuladores substituírem totalmente o uso de veículos reais nas formações ainda está a se comprovar, embora os resultados deste último estudo parecem frustrar tais expectativas, pelo menos para as formações iniciais. Por outro lado, o uso desse instrumento limitou-se, nas pesquisas investigadas, a treinar algumas competências específicas da condução profissional (Moraes, 2016).

No que tange às perspectivas metodológicas, as mais citadas são: análise da condução 
efetuada por meio de feedback, debriefing, análise dos erros e demonstração de exemplos em postura não punitiva (Blanco et al., 2011; Reep et al., 2013; Victor et al., 2011); aprendizagem progressiva e desenvolvimental (Bonch-Bruevich et al., 2010; Reep et al., 2013; Romoser \& Hirsch, 2012); baseada em repetições de exercícios e tarefas, com variação de cenários para o desenvolvimento de uma mesma competência (Aginaga-Garcia et al., 2013; Goode et al., 2013; Uhr et al., 2003); exercícios baseados em desempenhos realizados nos simuladores, indicando a customização da aprendizagem (Bonch-Bruevich et al., 2010; Tarr \& Whitmire, 2008). Os simuladores são também concebidos como instrumentos de verificação do desempenho e de levantamento de necessidade de treinamento (Reep et al., 2013; Tarr \& Whitmire, 2008; Victor et al., 2011), mas raramente citam-se seu uso de maneira mais lúdica, tais como baseado em jogos (Bonch-Bruevich et al., 2010), ou em formações envolvendo coletivos de análise (Lang, Diels, Grüneberg, \& Helmchen, 2011), o que reforça a ideia de que há potencialidades de usos ainda não exploradas.

\section{Papel dos Instrutores}

Não encontramos discussões sistemáticas sobre o papel que os instrutores devem ter nas formações desenvolvidas e/ou avaliadas, apesar de se reconhecer a importância de sua participação no sucesso da formação e dos usos de simuladores (Kihl et al., 2006; Reed et al., 2007). Para tanto, é importante seu entusiasmo frente ao instrumento, sua avaliação, registro e postura de receptividade e aceitação do desempenho dos alunos, seu contínuo feedback para os aprendizes, sua postura de confiança, apoio emocional, crítica sincera e capacidade de promover reflexões e engajamento individual e coletivo e seu estímulo para trocas críticas de experiências e aprendizagens comuns entre os aprendizes (Blanco et al., 2011; Kihl et al., 2007; Reed et al., 2007; Victor et al., 2011). Entretanto, não se tem muita clareza de como treiná-los (Morgan et al., 2011; Reep et al., 2013), já que o uso de instrutores não preparados para o uso de simuladores não necessariamente produz bons resultados nos treinamentos (Lang et al., 2011). Esses autores avaliaram também que a discussão (debriefing) é aliado metodológico fundamental para eficácia dos treinamentos.

Poucos estudos mencionam as dimensões da aprendizagem coletiva como elemento que o simulador propicia (Goode et al., 2013; Lang et al., 2011; Neukum et al., 2003). Alguns estudos ressaltam a importância dos instrutores como uma valiosa fonte de experiência para os novatos (Kihl et al., 2006; Morgan et al., 2011), mas essa dimensão recebe atenção apenas periférica. Saberes socialmente produzidos e partilhados, ainda que parte da atividade, tendem a não são objeto de intervenção nas formações profissionais com uso de simuladores.

\section{Algumas Análises}

A partir dessa revisão, podemos chegar a algumas primeiras conclusões: para uso generalizado dos simuladores, esbarra-se em problemas que devem ser considerados. Em primeiro lugar, os custos. Embora economicamente viáveis, já que os altos custos de aquisição e instalação do equipamento são compensados com economia de gastos com treinamento e seus benefícios (Morgan et al., 2011), o valor de investimento ainda é um empecilho. Em segundo lugar, muitos trabalhadores sofrem com o mal-estar de simulação durante as formações, reduzindo a eficiência do instrumento. Além disso, para o sucesso de seu uso, requer-se apoio institucional, métodos de formação bem integrados aos outros originariamente utilizados nas organizações e uma equipe de instrutores bem formada, elementos que não são facilmente mobilizados em organizações complexas.

Por outro lado, nos parece que os pressupostos teóricos-epistêmico-metodológicos dos usos dos simuladores também contribuem para essa defasagem. Pottier (2013) sugere, a partir de pesquisas com simuladores no campo da medicina, que diferentes abordagens teóricas sobre aprendizagem conduzem a distintos usos do instrumento. A partir da abordagem behaviorista preconiza-se o uso de simuladores como exercício para treinamento corporal por meio de 
repetição de seções de simulação, mediados por reforços positivos e negativos mobilizados pelo instrutor. A partir da perspectiva construtivista os simuladores se tornam meios de proposição de problemas que permitem assimilação e acomodação de novos saberes e competências aos esquemas prévios. Pela perspectiva social, que integra parcialmente a abordagem construtivista, preconizam-se simuladores como oportunidade: de partilha de saberes entre agentes, de reflexão coletiva sobre trabalho de equipe e sobre a aprendizagem cooperativa baseada na interdependência das diferentes profissões. Já por meio da perspectiva humanista, nas suas diferentes perspectivas, sugere-se que os simuladores, que usualmente comportam módulos de auto-avaliação, podem e devem ser utilizados de maneira autônoma pelos usuários, com ou sem apoio de tutores, propiciando aprendizagem autodirigida e calcada em fatores de motivação intrínseca, por meio de um vai-e-vem constante entre experimentação-reflexão-aplicação dos conceitos desenvolvidos. Além desses modelos citados pelo autor, verificamos que a Didática Profissional preconiza o uso de simuladores baseado na resolução de problemas, tal qual a abordagem construtivista, mas alia essa perspectiva o debriefing baseado na análise coletiva da atividade (Pastré, 2005), se aproximando de uma perspectiva mais social.

A partir dessa distinção, verificamos que as publicações e pesquisas que discutem a formação profissional dos transportes rodoviários, mesmo que não explicitado, pensam o uso de simuladores, em sua maioria, a partir de uma concepção behaviorista, sendo o treinamento comportamental e corporal os objetivos principais das formações (ver p. ex., Reep et al., 2013; Victor et al., 2011). Alguns poucos parecem mais afeitos a perspectivas construtivistas, quando sugerem cenários que visam evolução gradual e adaptativa dos cenários aos conhecimentos dos usuários (p. ex., Bonch-Bruevich et al., 2010; Romoser \& Hirsch, 2012). Muito raramente se fazem usos de simuladores conforme preconizado por perspectivas humanistas, que aventam a possibilidade de manipulação autônoma e independente do instrumento pelo aluno (Romoser \& Hirsch,
2012). Dois estudos sugerem treinamentos envolvendo formação em meio a pares, dentre os quais um deles preconiza formação envolvendo competências coletivas (Lang et al., 2011; Neukum et al., 2003), conforme preconiza a perspectiva social. Não há estudos que lançam mão da estratégia de resolução do problema, estratégia central tanto para a perspectiva construtivista ou da Didática Profissional, nem na análise da atividade. Destaca-se, também, que os cenários simulados, conforme as publicações citadas, não se referem, necessariamente, à atividade real de trabalho nem são produzidos a partir de análises da tarefa ou da atividade, salvo raras exceções (Mitsopoulos-Rubens, Lenné, \& Salmon, 2013a).

Observamos que se valoriza mais frequentemente os equipamentos que os desenhos dos treinamentos. Por exemplo, embora existam várias publicações propondo modelos de classificação de simuladores (p. ex., Brock et al., 2001; Eryilmaz, Tokmak, Cagiltay, Isler, \& Eryilmaz, 2014; Goode et al., 2013), poucas refletem sobre os pressupostos epistemológicos em formações (Lang et al., 2011). Uma vez que o uso de simuladores depende menos de seu desenvolvimento tecnológico que da compatibilidade entre as habilidades a serem treinadas e aquilo que o equipamento busca desenvolver (Brock et al., 2001), a primazia pelo instrumento, mais que pelo método de sua utilização, pode revelar subjacentemente uma noção de que a capacidade tecnológica enseja, em si, efeitos formadores. Isso indica também desqualificação de um debate que, na prática, pode contribuir para dificuldades ao se tentar aliar esses instrumentos aos métodos de formação já existentes (Mitsopoulos-Rubens, Lenné, \& Salmon, 2013b; Neukum et al., 2003; Reep et al., 2013). Por isso Mitsopoulos-Rubens et al. (2013b)_sugerem a retomada da discussão teórica sobre os usos dos simuladores como estratégia de melhoria de suas potencialidades.

Daí algumas outras conclusões provisórias: (a) não há grandes justificativas para se investir em simuladores (Grüneberg \& Schröder, 2012); (b) eles são instrumentos promissores, apesar de tudo (Moraes, 2016); (c) seus usos são limitados pelas referências teórico-epistemológica que as 
embasam; (d) há possibilidades de usos calcados em outros referenciais; e (e) são necessárias mais pesquisas sobre o assunto.

Os resultados dessa análise da literatura poderiam desestimular o uso dos simuladores, por não fornecer evidências suficientes de sua eficácia. Optamos por um caminho diferente: inspirados nas experiências francesas da Didática Profissional (Pastré, 2011), que utiliza a simulação como um dos pilares de suas práticas (Pastré, 2005), discutiremos os pontos 3 e 4 acima, sugerindo que os resultados encontrados nesta revisão talvez sejam mais problemas dos usos dos simuladores que de suas potencialidades, já que não se valorizou suficientemente as dimensões teórico-epistemológicas que poderiam propor novos caminhos para os usos do instrumento. Para realizar essa tarefa, lançaremos como ponto de apoio a perspectiva teórico-filosófica da $\mathrm{Er}$ gologia (Schwartz, 2012; Schwartz \& Durrive, 2010).

\section{Crítica Ergológica dos Usos Dos Simuladores}

Para a Ergologia (Schwartz, 2012), a atividade humana (seja ela de trabalho ou não) nunca é totalmente antecipável, ou previsível, já que em fazendo a pessoa inevitavelmente enseja transformações ao que se antecipou. Tais transformações decorrem dos esforços para se conciliar, por meio da própria atividade, as exigências e imposições prévias à ação - o que se denomina de normas antecedentes -, com as incongruências e incompatibilidades encontradas no meio, nos objetos, em outros homens com quem interage, nas normas e nas próprias condições pessoais, em meio a valores conflitantes pré-existentes à ação. E nesse esforço, aproveita-se para se tentar fazer do meio em que age, um meio seu, recentrando-o sobre si, sobre suas ações e necessidades (Schwartz, 2004; Schwartz $\&$ Echternacht, 2009).

Uma formação que se dá por meio do uso de simuladores não deixa de ser uma atividade, ainda que a atividade simulada não seja a mesma que aquela que se busca simular. Deduz-se daí que é impossível apreender totalmente as potencialidades e problemas do instrumento apenas por seu desenho, projeto, concepções e prescrições nele implícitas (em uma palavra, nas suas antecipações), mesmo que consideremos que suas qualidades e características influam, em algum modo, nas suas possibilidades de seu uso. As potencialidades dos simuladores se ampliam pelas reinvenções que se criam, com ele, pelos usuários durante suas utilizações cotidianas. Tais usabilidades imprevistas criadas pelos usuários podem sugerir novos empregos para os simuladores e resultados promissores dos treinamentos com tais instrumentos. Por outro lado, se verificarmos que nem os usos dos veículos regulares em situações de formação são comprovadamente eficazes para melhoria de desempenho ou redução de acidentes (Christie, 2001; Helman, Grayson, \& Parkes, 2010), não se pode responsabilizar apenas o instrumento pela qualidade das formações profissionais. Assim, o simulador ideal não é aquele mais tecnicamente próximo de um veículo real, seja em aparência, seja porque elicia nos usuários comportamentos semelhantes ao que se eliciam nos veículos reais, mas aquele que se relaciona funcionalmente aos objetivos do treinamento (Goode et al., 2013). Porém, a partir das considerações centrais da Ergologia, deve-se questionar, do ponto de vista dos aprendizes, se os objetivos das formações corresponderiam às necessidades impostas pela atividade real, ou às dos gestores e instrutores, geralmente centradas nas lógicas das antecipações que, como vimos, nunca são totalmente aplicadas.

Vimos alhures que aprendizes mais experientes criticam o realismo dos simuladores e que os inexperientes valorizam treinamentos em que há troca de experiência profissional com veteranos e instrutores (Kihl et al., 2006; Neukum et al., 2003). Esse aspecto sugerem 3 problemas existentes nestas formações, mas que valem para o conjunto das apresentadas na presente revisão: (a) não reconhecimento de qual atividade real e consequentemente quais saberes socialmente partilhados - simular nos simuladores e na formação, ou seja, um problema de incompatibilidade de objetivos da formação; (b) concepção teórico-epistemológica insuficiente sobre o que e quais seriam as competências e saberes em situ- 
ação de trabalho; e (c) como apreender uma atividade de trabalho. Discutiremos cada um desses pontos a partir dos pressupostos ergológicos, buscando apontar os limites dos simuladores e apresentar novas perspectivas. Apresentaremos, ao fim, algumas possibilidades de aplicação dos simuladores, advindas dessa reflexão.

\section{Não Reconhecimento da Atividade Real}

As pesquisas sobre formação profissional baseadas em análise de levantamento de necessidades ou de tarefas (Grüneberg \& Schröder, 2012; Mitsopoulos-Rubens et al., 2013a; Tarr \& Whitmire, 2008) ou sobre elaboração de simuladores e treinamentos (Dorn \& Stannard, 2006; Lindsey \& Barron, 2008) consideravam, quase exclusivamente, que as competências da simulação reduziam-se à condução em si, independente da condução simulada referir-se a uma situação de trabalho. Sem essa distinção, reduz-se a atividade de condução profissional ao comportamento de conduzir um veículo em uma situação tipificada de tráfego, deixando a atividade de trabalho em segundo plano (Hubault, 2011).

$\mathrm{Na}$ condução profissional encontram-se características específicas que influem diretamente no seu comportamento do condutor e no seu desempenho, entre as quais pressão para a realização da tarefa e exigências dos clientes e gestores (Hubault, 2011; Valot, 2007). Se isso não for considerado, subdimensiona-se o fim específico dessa condução - deslocamento de mercadorias e/ou pessoas com segurança - e as variabilidades dele consequentes, que impactam no desempenho e em seus desfechos. Mesmo na recente revisão de Goode et al. (2013) sobre a eficácia de simuladores não se observa tal distinção, o que leva os autores a comparar treinamentos simulados de quaisquer tipos de condução.

A falta de realismo dos simuladores em relação ao trabalho ou a algumas das tarefas que nele se executa (Brook-Carter et al., 2004; Kihl et al., 2006) pode ser sinal dessa defasagem: as questões e dificuldades enfrentadas no cotidiano de trabalho, a multiplicidades de tarefas a serem geridas para além da tarefa de condução em si, a monotonia, cansaço e mobilizações psicoafetivas no trabalho, e que jogam papel crucial nesta e em qualquer atividade profissional, são frequentemente desconsideradas. Os treinamentos até então pesquisados objetivam agregar competências aos trabalhadores baseados em um ideal previamente estabelecido, cuja referência tende a ser um comportamento abstrato de condutor, e não um trabalhador atuando em uma situação de trânsito. Diferente disso, poderíamos propor outros objetivos para tais treinamentos, tais como reflexão coletiva sobre a execução da atividade em situações reais e as dificuldades de se fazê-lo de modo econômico e seguro.

Ademais, se não há uma suficiente discussão teórico-epistemológica sobre a formação de competências para as situações de trabalho, menos ainda se discute sobre como elas se formariam e se transformariam na atividade. Nas pesquisas investigadas, pressupõem que é $n a$ atividade que uma competência adquirida em treinamentos para a atividade, será ou não demonstrada. Daí o desafio de se avaliar os simuladores e sua capacidade de transferência das competências nele desenvolvidas. Mas, se há competências que se desenvolvem também na atividade, as desenvolvidas em treinamentos nos simuladores não seriam necessariamente iguais. Assim, uma formação focando uma competência na atividade poderá requerer configurações diferentes das focadas para o que se antecipa à atividade. Como ilustração, observemos que a formação inicial de motoristas que prepara para o trânsito não garante o controle suficiente do veículo que será desenvolvido no trânsito. Semelhantemente, os simuladores que supostamente facilitam a transferência de aprendizagens por simularem situações de trânsito talvez não tenham sido suficientemente concebidos para simularem a atividade dos trabalhadores, restringindo-se, ainda, ao comportamento de condução. Retomar o treinamento da atividade real de trabalho requererá inserir nas formações com simuladores aspectos da atividade em situação e não apenas a condução e um tráfego tipificado.

\section{Concepção Teórico-Epistemológica Insuficiente sobre Competências}

Apesar de pesquisas sobre simuladores citarem alguns modelos de competência, talvez se 
devesse levar menos em conta as características em si das competências e mais a relação entre elas, o nível de experiência dos operadores e a situação singular que as mobiliza (Schwartz \& Durrive, 2010). As competências não são um comportamento em si, abstrato e idealizado, realizável independente de situações e replicável em qualquer circunstância (Schwartz, 1998). Para este autor, a competência é uma ação em situação que se manifesta por seis ingredientes que, articulados em maior ou menor grau, a depender da experiência profissional, das situações em tela e das relações coletivas ali existentes, possibilitam um agir competente na atividade, sempre dependente de situações concretas. Os seis ingredientes da competência são:

1. Domínio dos protocolos que organizam, orientam e/ou subsidiam as ações e que são calcados em saberes científicos, técnicos, gestionários, linguísticos, entre outros;

2. Incorporação do histórico de uma situação de trabalho, que além das tarefas e protocolos, se caracteriza também pela série de singularizações inevitáveis que ali se desenrolam, sejam elas problemas imprevistos, panes ou variabilidades dos sistemas sociotécnicos, e das produções de saberes desenvolvidos para enfrenta-las;

3. A capacidade de articulação da face protocolar (tarefas e outras antecipações) e da face singular (inesperada) de cada situação, de maneira a fazê-las circular sempre, colocando em sinergia os dois primeiros ingredientes;

4. A capacidade de fazer as arbitragens necessárias entre as diferentes exigências que se tem sobre si, por meio da mobilização de debate de valores ligado ao debate de normas, impostas e/ou instituídas na atividade; esse debate de valores e de normas se refere a uma renegociação permanente entre valores e normas de vida de quem age e os valores e normas de vida dos outros; trata-se, pois, de conseguir fazer valer, em meio a outros, suas próprias normas, tornando o meio em que se trabalho, o seu meio;

5. Ativação e duplicação do potencial da pessoa que se segue ao desenvolvimento da apropriação do meio como seu - ingrediente 4 - ampliando suas incidências, e dos valores a ela relacionado, sobre cada um dos ingredientes precedentes, de modo a por em sinergia as diversas faces de uma situação os protocolos, as singularizações, os debates de valores, ou seja, os ingredientes 1, 2, 3 e 4 - e o modo como cada um faz valer suas próprias normas de vida;

6. Aproveitamento das sinergias de competências, sempre coletivas e relativamente pertinentes, que estão presentes em cada situação de trabalho, para conseguir agir em meio, a partir e com outros (Schwartz \& Durrive, 2010).

Segundo essa perspectiva, uma competência, seja qual for, não é resultado de saberes definitivos, aprendidos isoladamente e que podem ser homogeneizados num mesmo plano de referência, mas uma rede de relações entre esses ingredientes que são, por si só, heterogêneos, mas que podem ser postos em sinergia, cada qual articulado singularmente em função das especificidades das demandas e situações, das diversas expertises em jogo e dos contextos em que se engendram. A competência em uma situação de trabalho é prejudicada quando não se é capaz de mobilizar adequadamente um desses ingredientes, mesmo se se realiza competentemente a atividade em outra situação. Vale ressaltar que tais ingredientes são frutos de uma história de cada trabalhador na atividade e requerem, inevitavelmente, o permanente trânsito entre as diversas facetas da atividade, seja ela a face protocolar, a dos valores ou a das singularizações. Ou seja, uma competência não é um saber objetificado que se transfere de uma situação de aula a uma realidade, mas o por em sinergia saberes protocolares e da história da situação, mobilizações pessoais e do coletivo, valores e suas repercussões em situação real.

Por exemplo: vimos anteriormente que nas pesquisas sobre simuladores, e como tais estudos apreendem as competências de condução, pouco se discute sobre como as dimensões coletivas da atividade manifestam-se nesta atividade e como poderiam ser mobilizadas por aquele instrumento. Na medida em que essas dimensões 
corresponderiam ao ingrediente $6^{\circ}$ (Schwartz, 1998), a falta de exercício suficiente dessas dimensões durante as situações de formação explicaria, em parte, porque os comportamentos ali aprendidos não são transferidos tal qual se espera, para as situações de trabalho. Essas mesmas considerações poderiam ser aplicadas a cada um dos outros ingredientes. Paradoxalmente, o uso de simuladores pode propiciar exercício de cada um desses ingredientes, por meio da variação de cenários para uma mesma competência, ideia também defendida por outros autores a partir de outros referenciais teóricos (Goode et al., 2013), incluindo aí as dimensões coletivas das competências. Daí, então, que uma formação baseada em ingredientes de competência ancoradas à situação de trabalho, poderia contribuir para ampliar os usos dos simuladores.

\section{Como Apreender uma Atividade de Trabalho}

Verifica-se importante definir o que se refere por atividade de trabalho. Reconhecendo a diferença entre o que se prescreve ao trabalhador e o que ele efetivamente executa, a tradição da Ergonomia francófona (Falzon, 2007) compreende a atividade para além do simples comportamento realizado das pessoas no trabalho. Ela também reconhece como parte da atividade as racionalidades operatórias dos trabalhadores, desenvolvidas a partir das experiências obtidas por meio da prática profissional, requerida pelas deficiências inevitáveis dos sistemas, das prescrições e das tarefas (Darses, Falzon, \& Munduteguy, 2007).

Desses princípios mais gerais e de outras contribuições teóricas e tomando como referência a atividade de trabalho, mas não se limitando a esta, Schwartz (2012) elabora uma démarche filosófica sobre a atividade humana, denominada de Ergologia, que a apreende a partir de quatro postulados:

1. A atividade nunca é mera execução do que socialmente se demanda ou exige, mas a inevitável recriação/regulação operatória desta demanda, mesmo que em ato quase imperceptível, mobilizado pelos acasos de situações sempre variáveis e variantes, pela partilha de reconcepções coletivas dos sabe- res, exigências e normas que socialmente se impõe e pelas necessidades e preferências pessoais, sempre singulares e impossíveis de desaparecerem totalmente no momento da ação;

2. Por essa distância sempre intransponível, torna-se impossível antecipar totalmente a atividade de outrem, uma vez que haverá sempre inventividades e recriações deste antecipado; daí a atividade ser a fonte da história humana;

3. É pelo corpo de cada um, corpo biológico, social, psicológico, provisório e sempre em reconstrução, que se executa a passagem entre o que se prescreve e o que se realiza;

4. Uma vez que na atividade sempre se faz diferentemente, há aí sempre escolhas sendo feitas, o que remete a atividade a um mundo de valores, preferências, disputas sociais e de história.

A partir desses postulados teóricos, verificamos que as pesquisas revisadas deixam de lado aspectos da atividade que justificam, parcialmente, os parcos efeitos das formações realizadas. Ao evidenciarmos tais aspectos, reconhecendo desde o inevitável do cotidiano, ao corpo histórico e social, abrimos possibilidades de análise e intervenção em pesquisas futuras.

\section{Perspectiva Ergológicas para Usos de Simuladores}

A partir das análises ergológicas realizadas, verificam-se, a título de conclusão, algumas perspectivas para a formação de profissionais dos transportes terrestres com o uso simuladores. A mobilização de tais aspectos podem contribuir para pesquisas e práticas de formação de condutores profissionais com uso de simuladores, a despeito de seu uso ainda não estar prevista em resoluções nacionais sobre o assunto:

1. Desenvolvimento de simuladores e formações que simulem a atividade que se busca desenvolver, mais que simplesmente o comportamento de um veículo no trânsito;

2. Formações coletivas compostas por trabalhadores com mais e com menos experiência, ampliando as possibilidades de uso de 
instrutores sem experiência profissional, contrário ao que alguns propuseram (Brock et al., 2001; Grüneberg \& Schröder, 2012). Com formações coletivas como essa, reduz-se parcialmente a exigência de saberes profissionais por parte dos instrutores, ficando sua tarefa mais para mediar debates, promover entusiasmo, manter postura adequada e mobilizar adequadamente teorias, métodos e instrumentos de formação exigidos pelos simuladores;

3. Formações como possibilidade de debate e relação entre saberes científicos e os constituídos na experiência profissional. $\mathrm{O}$ uso de instrutores sem experiência na profissão treinada contribui para essa direção, se a formação envolve um coletivo;

4. Os cenários não devem constituir-se apenas em torno do desenvolvimento de competências singulares, mas também em torno dos ingredientes das competências (Schwartz, 1998) que propiciem análises (debriefing) que ampliem a capacidade de reflexão dos envolvidos nessas atividades. Mesmo em formação inicial, mais que um simples treinamento corporal, os simuladores devem servir como mediadores dos valores e saberes partilhados pelos coletivos. Considera-se aqui, inclusive, que o próprio gesto profissional tem sua raiz socialmente produzida e transmissível (Clot, 2010);

5. Criação de cenários que mobilizam a análise dos valores subjacentes às atividades. Entre pistas possíveis, sugere-se cenários com tarefas lúdicas e/ou desafiadoras, como jogos, que mobilizam nos usuários emoções que se aproximam das vivenciadas em situação reais de trabalho. Da angústia pelo prazo, ao prazer da condução, uma atividade de trabalho nunca é realizada totalmente apática, daí que um trabalho não ser nunca neutro para a saúde (Dejours, 2012); e

6. Desenhos de formações calcadas nas reais necessidades da atividade dos trabalhadores e não na mera análise da tarefa (Mitsopoulos-Rubens et al., 2013a). Sem ser um pacote fechado de saberes a se desenvolver, tal perspectiva de formação, que envolve inevitavelmente o usuário final, pode contribuir para o desenvolvimento das formações e do próprio instrumento (Romoser \& Hirsch, 2012).

Finalmente, alertamos quanto à tentação de se buscar reduzir índices de acidentes de trabalho calcado apenas em formação dos profissionais. Essa expectativa pode limitar as análises sobre os fatores relacionados aos acidentes, que envolvem outras causas, além das competências e características de personalidade dos condutores. Além disso, há indícios da limitada eficácia de treinamentos para a formação de condutores e seus efeitos na redução de acidentes de transportes (Christie, 2001; Helman et al., 2010), além de que determinadas práticas de gestão parecem ser mais eficientes no controle dos acidentes que os treinamentos (Christie, 2001). Nessa direção, o treinamento deve ser considerado apenas como mais um fator que contribui para a segurança nas estradas.

Pesquisas futuras poderão verificar em que medidas essas sugestões apontadas terão efeitos superiores aos verificados até o momento. De todo modo, demonstramos que os usos dos simuladores, quando ancorados em reflexões teórico-epistemológicas e metodológicas, ampliam suas possibilidades, o que deve ser levado em conta pelos setores responsáveis pela produção de resoluções que regulam a formações profissional de condutores.

\section{Referências}

Abbad, G. S., Zerbini, T., Carvalho, R. S., \& Meneses, P. P. M. (2006). Planejamento instrucional em TD\&E. In J. E. Borges-Andrade, G. S. Abbad, \& L. Mourão (Eds.), Treinamento, desenvolvimento e educação em organizações e trabalho: Fundamentos para a gestão de pessoas (pp. 289-321). Porto Alegre, RS: Artmed.

Aginaga-Garcia, J., Iriarte-Goni, X., Pintor-Borobia, J. M., Ros-Ganuza, J., \& San Miguel-Indurain, J. (2013). Implantación de un simulador para la formación de conductores de carretillas elevadoras. Dyna, 88(5), 574-580. doi:10.6036/5581 
Blanco, M., Hickman, J. S., Hanowski, R. J., \& Morgan, J. F. (2011). The Commercial Driver. In D. L. Fisher, M. Rizzo, J. K. Caird, \& J. D. Lee (Eds.), Handbook of driving simulation for engineering, medicine and psychology (pp. 241256). Boca Raton, FL: CRC.

Bonch-Bruevich, V. V., Kremez, A. S., \& Chirkov, B. P. (2010). Profession-relevant skills development using psychophysiological trainer. Automation and Remote Control, 71(7), 1461-1464. doi:10.1134/s0005117910070222

Brock, J. F., Jacobs, C., Van Cott, H., McCauley, M., \& Norstrom, D. M. (2001). Simulators and bus safety: Guidelines for acquiring and using transit bus operator driving simulators. Washington, DC: Transportation Research Board.

Brook-Carter, N., Luke, T., \& Parkes, A. (2004, October 4-6). Evaluating the use of a truck simulator for training drivers in safe and fuel efficient driving. Paper presented in European Transport Conference, Strasbourg, France.

Castro, M. N. M., \& Ferreira, L. D. V. (2006). TD\&E a distância: Múltiplas mídias e clientelas. In J. E. Borges-Andrade, G. S. Abbad, \& L. Mourão (Eds.), Treinamento, desenvolvimento e educação em organizações e trabalho: Fundamentos para a gestão de pessoas (pp. 322-339). Porto Alegre, RS: Artmed.

Christie, R. (2001). The effectiveness of driver training as a road safety measure: an international review of the literature. Paper presented in Road Safety Research, Policing and Education Conference, Melbourne, Australia.

Clot, Y. (2010). Poder de agir. Belo Horizonte, MG: FabreFactum.

Darses, F., Falzon, P., \& Munduteguy, C. (2007). Paradigmas e modelos para a análise cognitiva das atividades finalizadas. In P. Falzon (Ed.), Ergonomia (pp. 155-173). São Paulo, SP: Blucher.

Dejours, C. (2012). Trabalho vivo (Vol. 1-2). Brasília, DF: Paralelo 15.

Directive 2003/59/EC of the European Parliament and of the Council of 15 July. (2003, September 10). Official Journal of the European Union, L $226 / 4$.

Dorn, L., \& Stannard, J. (2006). Simulator Performance Differences between experienced and novice bus drivers [Special issue]. Advances in Transportation Studies, 45-52.
Edson, J. M. (2003). Simulator-based equipment operator training. Paper presented in $10^{\text {th }}$ AASHTO-TRB Maintenance Management Conference, Duluth, MN.

Eryilmaz, U., Tokmak, H. S., Cagiltay, K., Isler, V., \& Eryilmaz, N. O. (2014). A novel classification method for driving simulators based on existing flight simulator classification standards. Transportation Research Part C: Emerging Technologies, 42, 132-146. doi:10.1016/j.trc.2014.02.011

Falzon, P. (Ed.). (2007). Ergonomia. São Paulo, SP: Edgar Blucher

Goode, N., Salmon, P. M., \& Lenné, M. G. (2013). Simulation-based driver and vehicle crew training: Applications, efficacy and future directions. Applied Ergonomics, 44(3), 435-444. doi:10.1016/j.apergo.2012.10.007

Grüneberg, U., \& Schröder, A. (2012). Status of simulator training for professional drivers in Europe. Project SIMTEB: Simulator-based training for European bus drivers. Dortmund, German: European Comunity.

Helman, S., Grayson, G. B., \& Parkes, A. M. (2010). How can we produce safer new drivers? A review of the effects of experience, training and limiting exposure on the collision risk of new drivers. TRL INSIGHT REPORT(S005).

Hubault, F. (Ed.). (2011). Activité de conduite, activité de travail. Toulouse, France: Octares.

Kihl, M., Herring, D., Wolf, P., Finn, M., \& Yang, P. (2007). Snowplow Simulator Training Evaluation: Potential fuel and drivetrain maintenance cost reduction. Phoenix, AZ: Arizona Department of Transportation.

Kihl, M., Herring, D., Wolf, P., McVey, S., \& Kovuru, V. (2006). Snowplow Simulator Training Evaluation. Phoenix, AZ: Arizona Department of Transportation.

Lang, B., Diels, C., Grüneberg, U., \& Helmchen, G. (2011, November 29-30). Lessons Learned from Simulator-Based Training for Bus Drivers. Paper presented in International Driver Behaviour and Training Conference, Paris.

Lindsey, J. T., \& Barron, A. E. (2008). Effects of Simulation on Emergency Vehicle Drivers' Competency in Training. Prehospital and Disaster Medicine, 23(4), 361-368.

Michon, J. A. (1985). A critical view of driver behavior models: What do we know, what 
should we do? In L. Evans \& R. C. Schwing (Eds.), Behavior and traffic safety (pp. 485-520). New York: Plenum Press.

Mitsopoulos-Rubens, E., Lenné, M. G., \& Salmon, P. M. (2013a). Driver simulation-based training of heavy vehicle operators: Targeted task analysis and considerations for training design. Paper presented in $7^{\text {th }}$ International Driving Symposium on Human Factors in Driver Assessment, Training, and Vehicle Design, New York.

Mitsopoulos-Rubens, E., Lenné, M. G., \& Salmon, P. M. (2013b). Effectiveness of simulator-based training for heavy vehicle operators: What do we know and what do we still need to know? Paper presented in 2013 Australasian Road Safety Research, Policing \& Education Conference, Brisbane, Australia.

Mooren, L., Grzebieta, R., Williamson, A., Olivier, J., \& Friswell, R. (2014). Safety management for heavy vehicle transport: A review of the literature. Safety Science, 62, 79-89. doi:10.1016/j.ssci.2013.08.001

Moraes, T. D. (2016). O uso de simuladores em atividades de formação para profissionais dos transportes rodoviários. Ciencia \& Trabajo, 18(55), 48-57. doi:10.4067/S0718-24492016000100009

Morgan, J. F., Tidwell, S. A., Medina, A., Blanco, M., Hickman, J. S., \& Hanowski, R. J. (2011). Commercial Motor Vehicle Driving Simulator Validation Study: Phase II. Washington, DC: US Departament of Transportation, Federal Motor Carrier Safety Administration.

Navarrete, J. A. R., Urquiza, E. M., Hernandez, M. V., \& Madrid, M. M. (2004). Generalidades sobre el entrenamiento de conductores y el desarrollo de simuladores de manejo. Sanfadila, Mexico: Instituto Mexicano del Transporte.

Neukum, A., Lang, B., \& Krueger, H.-P. (2003, October 8-10). A simulator-based training for emergency vehicle driving. Paper presented in Proceedings of the Driver Simulation Conference, North America, Dearborn, MI.

Pastré, P. (Ed.). (2005). Apprendre par la simulation: de l'analyse du travail aux apprentissages professionnels. Toulouse, France: Octares.

Pastré, P. (2011). La didactique professionnelle: approche anthropologique du développement chez les adultes. Paris: Presses Universitaires de France.
Pottier, P. (2013). Théories de l'apprentissage et simulation: le point de vue du professionnel de santé-enseignant. In S. Boet, J.-C. Granry, \& G. Savoldelli (Eds.), La simulation en santé: de la théorie à la pratique (pp. 15-24). Paris: Springer Paris.

Rasmussen, J. (1983). Skills, rules, and knowledge; signals, signs, and symbols, and other distinctions in human performance models. Systems, Man and Cybernetics, IEEE Transactions on, SMC-13(3), 257-266. doi:10.1109/TSMC.1983.6313160

Reed, N., Parkes, A. M., Peacock, C., Lang, B., \& Rehm, L. (2007). SCOTSIM: An evaluation of the effectiveness of two truck simulators for professional driver training. Scotland, UK: Enterprise, Transport \& Lifelong Learning Departament.

Reep, A., Staes, L., \& Perk, V. (2013). A qualitative analysis of bus simulator training on transit incidents: A case study in Florida. Jacksonville, FL: National Center for Transit Research.

Resolução Conselho Nacional de Trânsito no 493 de 25 de junho. (2014, 06 jun.). Diário Oficial da União.

Resolução Conselho Nacional de Trânsito $n^{\circ}$ 543, de 15 de julho. (2015, 20 jul.). Diário Oficial da União.

Romoser, M. R. E., \& Hirsch, P. (2012). From lab to real life: A case study in the deployment of advanced driving simulator-based training systems. Proceedings of the Human Factors and Ergonomics Society Annual Meeting, 56(1), 2527-2531. doi:10.1177/1071181312561525

Santana, V., Nobre, L., \& Waldvogel, B. C. (2005). Acidentes de trabalho no Brasil entre 1994 e 2004: Uma revisão. Ciência \& Saúde Coletiva, 10(4), 841-855. doi:10.1590/S141381232005000400009

Schwartz, Y. (1998). Os ingredientes da competência: Um exercício necessário para uma questão insolúvel. Educação \& Sociedade, 19(65), 101140.

Schwartz, Y. (2004). Circulações, dramáticas, eficácias da atividade industriosa. Trabalho, Educação e Saúde, 2(1), 33-55. doi:10.1590/ S1981-77462004000100004

Schwartz, Y. (2012). Expérience et connaissance du travail. Paris: Les éd. sociales. 
Schwartz, Y., \& Durrive, L. (Eds.). (2010). Trabalho e ergologia: Conversas sobre a atividade humana. Niterói, RJ: Editora da Universidade Federal Fluminense.

Schwartz, Y., \& Echternacht, E. (2009). Le corps-soi dans les milieux de travail : comment se spécifie sa compétence à vivre? Corps, 1(6), 31-37. doi:10.3917/corp.006.0031

Strayer, D. L., \& Drews, F. A. (2003). Simulator training improves driver efficiency: Transfer from the simulator to the real world. Paper presented in $2^{\text {nd }}$ International Driving Symposium on Human Factors in Driver Assessment, Training and Vehicle Design, Salt Lake City, UT.

Tarr, R. (2005). Simulation as a Tool for Enhancing Commercial Driver Skills: A Systematic Approach to Tailored Training. Paper presented in Driving Simulation Conference, Orlando, FL.

Tarr, R., \& Whitmire, J. D. (2008). Simulation as a Tool for Enhancing Commercial Driver Skills and Recertification: Follow-On to the Virtual Check Ride System. Tallahassee, FL: Florida Department of Transport, Motor Carrier Compliance Office.
Uhr, M. B. F., Felix, D., Williams, B. J., \& Krueger, H. (2003, October 8-10). Transfer of Training in an Advanced Driving Simulator: Comparison between Real World Environment and Simulation in a Manoeuvring Driving Task. Paper presented in DSC North America 2003 Proceedings, Dearborn, MI.

Valot, C. (2007). O transporte, a segurança e a ergonomia. In P. Falzon (Ed.), Ergonomia (pp. 573-586). São Paulo, SP: Edgar Blucher.

Victor, T., Hickman, J., Camden, M., Jarlengrip, J., Larsson, C., Morgan, J., ...Toole, L. (2011). U34: Driver Distraction: An Inattention-Mitigation Component for Behavior-Based Safety Programs in Commercial Vehicle Operations (IM-BBS). Washington, DC: National Transportation Research Center.

Recebido: 25/02/2016

$1^{a}$ revisão: $15 / 08 / 2016$

Aceite final: 16/08/2016 